\title{
Composição Corporal e Requisitos Líquidos e Dietéticos de Macroelementos Minerais de Bovinos Nelore Não-Castrados ${ }^{1}$
}

\author{
Antonia Sherlânea Chaves Véras ${ }^{2}$, Sebastião de Campos Valadares Filho ${ }^{3}$,José Fernando \\ Coelho da Silva ${ }^{4}$, Mário Fonseca Paulino ${ }^{3}$, Paulo Roberto Cecon ${ }^{5}$, Rilene Ferreira Diniz Valadares ${ }^{6}$, \\ Marcelo de Andrade Ferreira ${ }^{2}$, Pedro Veiga Rodrigues Paulino ${ }^{7}$, Cleiton Vieira Rocha ${ }^{8}$
}

RESUMO - Utilizaram-se 30 animais Nelore, não-castrados, com idade e peso médios de 20 meses e $330 \mathrm{~kg}$, para avaliar os efeitos da inclusão de concentrado sobre os requisitos líquidos e dietéticos de cálcio (Ca), fósforo (P), magnésio (Mg) e potássio (K). Após um período de adaptação (40 dias), cinco animais foram abatidos para servir como referência e os 25 restantes, distribuídos em cinco tratamentos $(25,0 ; 37,5 ; 50,0 ; 62,5$; e 75,0\% de concentrado, na MS), em delineamento inteiramente casualizado, com cinco repetições. O peso de abate foi estabelecido como $450 \mathrm{~kg}$. Ajustaram-se equações de regressão do logaritmo do conteúdo corporal de Ca, P, Mg ou K, em função do logaritmo do peso do corpo vazio (PCVZ), para cada nível de concentrado nas dietas. Derivando-se as referidas equações, obtiveram-se os requisitos líquidos para ganho de 1 kg de PCVZ (GPCVZ). Para estimativa dos requisitos dietéticos dos elementos inorgânicos avaliados, utilizaram-se os coeficientes médios de absorção estimados previamente, de 53,13; 37,33; 39,62; e 65,54\%, para Ca, $\mathrm{P}, \mathrm{Mg}$ e $\mathrm{K}$, respectivamente. Os requerimentos líquidos dos elementos minerais diminuíram com o aumento do PCVZ dos animais. Para um animal de $400 \mathrm{~kg}$ de PV, as exigências líquidas foram de 8,$18 ; 7,53 ; 0,21 ;$ e 2,77 g/kgGPCVZ, respectivamente, para Ca, P, Mg e K. Já os requisitos dietéticos para um bovino com este mesmo peso foram de 15,$40 ; 20,17 ; 0,53 ;$ e 4,23 g/kg GPCVZ, respectivamente, para a mesma seqüência de minerais. Os requisitos de $\mathrm{Ca}, \mathrm{P}, \mathrm{Mg}$ e K diferiram dos preconizados pelos sistemas britânico e americano de exigências nutricionais.

Palavras-chave: composição corporal, exigências, macroelementos minerais, Nelore

\section{Body Composition and Liquid and Dietary Requirements of Macrominerals of Nellore Bulls}

\begin{abstract}
Thirty Nellore bulls, averaging 20 months of age and $330 \mathrm{~kg}$, were used to evaluate the effects of concentrate inclusion on the liquid and dietary requirements of calcium (Ca), phosphorus (P), magnesium (Mg) and potassium (K). After a period of adaptation ( 40 days), five animals were slaughtered as reference and the 25 remaining were allotted to five treatments $(25.0,37.5,50.0,62.5$, and $75.0 \%$ of concentrate, in DM), in a completely randomized design, with five replicates. The slaughter weight was established as $450 \mathrm{~kg}$. Regression equations of the logarithm of $\mathrm{Ca}, \mathrm{P}, \mathrm{Mg}$ or $\mathrm{K}$ body content were fitted in function of the logarithm of the empty body weight (EBW), for the concentrate levels in the diets. By deriving these equations, liquid requirements for gain of $1 \mathrm{~kg} \mathrm{EBW}$ (EBWG) were obtained. The previous average estimated coefficients of absorption, of 53.13, $37.33,39.62$, and $65.54 \%$, for $\mathrm{Ca}, \mathrm{P}, \mathrm{Mg}$ and $\mathrm{K}$, respectively, were used to determine the macrominerals dietary requirements. The macrominerals liquid requirements decreased as EBW increased. For a $400 \mathrm{~kg} \mathrm{LW}$ animal, the liquid requirements were 8.18, 7.53, .21 , and $2.77 \mathrm{~g} / \mathrm{kg}$ EBWG, respectively, for $\mathrm{Ca}, \mathrm{P}, \mathrm{Mg}$ and $\mathrm{K}$. The dietary requirements for a $400 \mathrm{~kg} \mathrm{LW}$ bovine were $15.40,20.17$, .53 , and $4.23 \mathrm{~g} / \mathrm{kg}$ EBWG, respectively, for the same macrominerals sequence. The $\mathrm{Ca}, \mathrm{P}, \mathrm{Mg}$ and $\mathrm{K}$ requirements differed from the British and American nutritional requirements systems.
\end{abstract}

Key Words: body composition, requirements, macrominerals, Nellore

\footnotetext{
1 Parte da tese de Doutorado do primeiro autor, parcialmente financiada pela FINEP

2 Professor do DZ/UFRPE. E.mail: sherlanea@uol.com.br

3 Professor do DZ/UFV.

4 Professor do LZNA-CCTA/UENF.

5 Professor do DINF/UFV.

6 Professor do DMV/UFV

7 BolsistaPIBIC/DZ/UFV.

8 Aluno de Graduação em Zootecnia /UFV.
} 


\section{Introdução}

Embora representem apenas 4\% do peso corporal dos animais, os minerais estão presentes em proporções variáveis em todos os tecidos e exercem funções vitais no organismo, com reflexos no desempenho animal (DAYRELL, 1993).

Segundo NICODEMO (1999), a ingestão contínua de dietas deficientes ou desequilibradas em minerais essenciais pode desenvolver lesões bioquímicas, prejudicando as funções biológicas e levar a desordens estruturais que variam com o mineral, a intensidade e duração da deficiência, a idade, a condição sexual e a espécie animal.

No caso de ruminantes, um fornecimento adequado de minerais é importante para otimização da atividade microbiana no rúmen (NATIONAL RESEARCH COUNCIL - NRC, 1996), com sua deficiência produzindo impacto negativo sobre o crescimento microbiano, podendo induzir, ou não, redução da digestibilidade dos alimentos, dependendo da severidade da carência mineral (LENG, 1990; SPEARS, 1994) e da disponibilidade do mineral (SPEARS, 1994).

Os requisitos nutricionais de macroelementos minerais são geralmente estimados pelo método fatorial. A determinação das exigências líquidas para crescimento e engorda depende da deposição do mineral no corpo do animal que se verifica a diferentes idades e pesos, geralmente obtida com o abate dos animais e posterior determinação do conteúdo de minerais nos tecidos corporais (COELHO DA SILVA, 1995).

Aos requerimentos para ganho devem ser acrescidas as quantidades de minerais necessárias para mantença, que são relativas às necessidades para atender as perdas inevitáveis do corpo, ou seja, as secreções endógenas. A exigência líquida total é obtida pela soma dos requisitos para mantença e ganho, que, após correção pelo coeficiente de absorção do elemento inorgânico no trato digestivo do animal, resulta na exigência dietética do animal (ARC, 1980; COMMONWEALTH SCIENTIFIC AND INDUSTRIAL RESEARCH ORGANIZATION CSIRO, 1990; NRC, 1996).

O cálcio $(\mathrm{Ca})$ e o fósforo $(\mathrm{P})$ representam, juntos, cerca de $70 \%$ do total de elementos inorgânicos do corpo, segundo o NRC (1996) e, aproximadamente $99 \%$ do Ca no corpo é encontrado no esqueleto, sendo que o músculo contém $100 \mathrm{mg} / \mathrm{kg}$ de peso do material in natura, a gordura não contém $\mathrm{Ca}$, e os ossos, de 100 a 200 g/kg. Cerca de $80 \%$ do P estão presentes no esqueleto e esta fração está intimamente associada ao $\mathrm{Ca}$ presente nos ossos na forma de hidroxiapatita. Os ossos contêm de 50 a $100 \mathrm{~g}$ de P/kg, os músculos de 2 a $3 \mathrm{~g} / \mathrm{kg}$ e a gordura contém quantidades mínimas, na forma de fosfolipídios (AGRICULTURAL AND FOOD RESEARCH COUNCIL - AFRC, 1991; COELHO DA SILVA, 1995).

Os ossos contêm $2 \mathrm{~g}$ de magnésio $(\mathrm{Mg})$ por $\mathrm{kg}$ de material fresco e a relação Ca: $\mathrm{Mg}$ é 55:1, sendo que $70 \%$ do $\mathrm{Mg}$ presente no corpo estão nos ossos, os músculos contêm $190 \mathrm{mg}$ de $\mathrm{Mg} / \mathrm{kg}$ (COELHO DA SILVA, 1995), enquanto $1 \%$ está associado aos espaços extracelulares (CSIRO, 1990).

Já em relação ao potássio (K), este mineral está presente nos ossos em quantidades mínimas, que correspondem a valores inferiores a $50 \mathrm{mg}$ de $\mathrm{K} / \mathrm{kg}$, os músculos contêm $4 \mathrm{~g} / \mathrm{kg}$, os tecidos nervosos e secretórios, 3,5 g/ $\mathrm{kg}$, e o soro e fluidos, $200 \mathrm{mg} / \mathrm{kg}$ (COELHO DA SILVA, 1995). A CSIRO (1990) relatou menores concentrações de $\mathrm{K}$ no corpo de animais velhos ou gordos, porque o músculo contém mais K que outros tecidos. O ARC (1980) estimou os requisitos líquidos de $\mathrm{Mg}$ e $\mathrm{K}$ em 0,45 e $2 \mathrm{~g} / \mathrm{kg}$ de GPCVZ, respectivamente, independente do PCVZ do animal.

A retenção de minerais depende da composição do ganho. Maiores deposições de gordura reduzem as deposições de elementos inorgânicos e, consequentemente, seus requerimentos pelos animais, já que as concentrações de minerais no tecido adiposo são menores que nos músculos e nos ossos. Portanto, fatores como condição sexual, grupo genético, peso e idade dos animais influenciam os requerimentos de minerais.

O ARC (1980), analisando resultados experimentais relativos a animais com peso de corpo vazio (PCVZ) variando entre 75 e $500 \mathrm{~kg}$, concluiu não haver queda nas concentrações de $\mathrm{Ca}$ e $\mathrm{P}$, com o aumento do PCVZ, adotando 14 e 8 g, respectivamente, tanto por kg de PCVZ, quanto por kg de ganho de PCVZ. A CSIRO (1990) também adotou os valores do ARC (1980). Contudo, era esperada queda nas concentrações dos minerais com o aumento da gordura corporal, pois os depósitos de gordura não contêm Ca e os triglicerídios dos depósitos adiposos contêm apenas quantidades negligenciáveis de $\mathrm{P}$ (COELHO DA SILVA, 1995; FONTES 1995).

O AFRC (1991), reexaminando o modelo adotado pelo ARC (1980), propôs nova metodologia para determinação dos requisitos de $\mathrm{Ca} \mathrm{e} \mathrm{P}$, introduzindo o peso à maturidade e o peso vivo (PV), ao invés do 
1108 Rev. bras. zootec.

PCVZ. Assim, foi possível verificar decréscimo na deposição de Ca e P, à medida que o PV se eleva.

Segundo o AFRC (1991), para que ocorra desenvolvimento normal dos ossos, além de suprimento adequado de minerais, são necessários níveis adequados de proteína e energia. O NRC (1996) estimou os requerimentos líquidos de $\mathrm{Ca}$ e $\mathrm{P}$ para ganho de peso em função do ganho diário de proteína. Para Mg e K, recomendou médias de 0,10 e 0,6\% na MS da dieta, respectivamente, como requisitos dietéticos.

Ainda são poucas as informações disponíveis no Brasil sobre os requerimentos de macroelementos minerais para bovinos de corte e, segundo COELHO DA SILVA (1995), os resultados são um tanto quanto desuniformes.

O presente trabalho foi conduzido objetivando avaliar o efeito de diferentes níveis de concentrado sobre a composição corporal e as exigências líquidas e dietéticas dos macroelementos inorgânicos $(\mathrm{Ca}, \mathrm{P}$, Mg e K), de bovinos Nelore não-castrados.

\section{Material e Métodos}

O local do experimento, as instalações, as dietas (proporção dos ingredientes e composição), o sistema de alimentação, o manejo utilizado, o ensaio de digestibilidade, os procedimentos de abate, as coletas de amostras, as análises químicas dos alimentos e as análises estatísticas foram descritos por VÉRAS et al. (2000a,b).

Foram utilizados 30 bovinos Nelore, não-castrados, com peso médio inicial de $330 \mathrm{~kg}$ e idade média de 20 meses. Após um período de adaptação de 40 dias, cinco animais foram abatidos para servir como referência para a estimativa do peso do corpo vazio (PCVZ) e da composição corporal iniciais dos 25 remanescentes. Estes foram distribuídos em cinco tratamentos com diferentes níveis de concentrado na matéria seca das dietas $(25,0 ; 37,5 ; 50,0 ; 62,5$; e $75,0 \%$ ), em delineamento inteiramente casualizado, com cinco repetições.

Os conteúdos corporais de macroelementos minerais foram determinados em função das concentrações percentuais destes nos órgãos, nas vísceras, no couro, no sangue, na cauda, na cabeça, nos pés (gordura e ossos) e nos constituintes separados (gordura, músculos e ossos) da seção entre a $9^{\mathrm{a}}$ e $11^{\mathrm{a}}$ costelas da meia-carcaça esquerda (seção HH), esta última representando a composição da carcaça.

A solução mineral para determinação dos macroelementos minerais foi preparada por via úmida. Após as devidas diluições, o teor de $\mathrm{P}$ foi determinado por colorimetria, os de $\mathrm{Ca}$ e $\mathrm{Mg}$, em espectrofotômetro de absorção atômica, e os de K, em espectrofotômetro de chama.

Para predição das quantidades líquidas de macroelementos inorgânicos retidos no corpo dos animais de cada tratamento, e para todos os tratamentos em conjunto, utilizaram-se equações de regressão do logaritmo do conteúdo corporal de $\mathrm{Ca}, \mathrm{P}$, $\mathrm{Mg}$ ou K, em função do logaritmo do PCVZ, conforme o seguinte modelo:

$$
\mathrm{Y}=\mathrm{a}+\mathrm{bX}+\mathrm{e}
$$

em que: $\mathrm{Y}=$ logaritmo do conteúdo total do macroelemento inorgânico $(\mathrm{kg})$, retido no corpo vazio; $a=$ efeito da média (intercepta); $b=$ coeficiente de regressão do logaritmo do conteúdo do macroelemento inorgânico, em função do logaritmo do PCVZ; $\mathrm{X}=$ logaritmo do PCVZ; e = erro aleatório.

Para cada tratamento, as equações foram construídas adicionando-se os valores relativos aos dos animais referência.

Derivando-se as equações de predição do conteúdo corporal de macroelementos inorgânicos em função do logaritmo do PCVZ foram obtidas as equações de predição das exigências líquidas de $\mathrm{Ca}$, $\mathrm{P}, \mathrm{Mg}$ e K para ganho de um $\mathrm{kg}$ de PCVZ, do tipo:

$$
\mathrm{Y}^{\prime}=\mathrm{b} \cdot 10^{\mathrm{a}} \cdot \mathrm{X}^{\mathrm{b}-1}
$$

em que: $Y^{\prime}=$ exigência líquida do macroelemento inorgânico; a e b = intercepta e coeficiente de regressão, respectivamente, das equações de predição dos conteúdos corporais de macroelementos inorgânicos; $\mathrm{e} ; \mathrm{X}=\mathrm{PCVZ}(\mathrm{kg})$.

Os requisitos dietéticos dos elementos minerais foram estimados a partir da relação entre os requerimentos líquidos obtidos por meio das equações em conjunto obtidas neste trabalho e os respectivos coeficientes médios de absorção aparente, para cada elemento mineral, obtidos por VÉRAS et al. (2000a).

Para predição do PCVZ a partir do PV foi utilizada a equação geral obtida por VÉRAS et al. (2000b), ajustada para todos os dados: $\mathrm{PCVZ}=-46,262+0,9911$ $\mathrm{PV} ; \mathrm{r}^{2}=0,94$. $\mathrm{O}$ fator para a conversão das exigências para ganho de PCVZ em exigências para ganho de PV obtido a partir desta equação foi igual a 1 .

\section{Resultados e Discussão}

Na Tabela 1 são apresentados os parâmetros das equações de regressão do logaritmo dos conteúdos de cálcio $(\mathrm{Ca})$, fósforo $(\mathrm{P})$, magnésio $(\mathrm{Mg})$ e potássio 
Tabela 1 - Parâmetros das equações de regressão do logaritmo dos conteúdos $(\mathrm{kg})$ de cálcio, fósforo magnésio e potássio no corpo vazio, em função do logaritmo do peso do corpo vazio $(\mathrm{kg})$ de bovinos Nelore, para os diferentes níveis de concentrado (NC) na ração, e em conjunto (CONJ)

Table 1 - Parameters of the logarithm regression equations of calcium, phosphorus, magnesium and potassium contents $(\mathrm{kg})$ in the empty body, on the empty weight logarithm $(\mathrm{kg})$ of Nellore bulls, for the different levels of concentrate in the diet, and in overall

\begin{tabular}{lccc}
\hline NC $(\%)$ & \multicolumn{3}{c}{$\begin{array}{c}\text { Parâmetros } \\
\text { Parameters }\end{array}$} \\
\cline { 2 - 4 } & $\begin{array}{c}\text { Intercepta (a) } \\
\text { Intercept }\end{array}$ & $\begin{array}{c}\text { Coeficiente (b) } \\
\text { Coefficient }\end{array}$ & $\mathrm{r}^{2}$ \\
\hline \multicolumn{3}{c}{$\begin{array}{c}\text { Cálcio(kg) } \\
\text { Calcium } \\
\text { 25 }\end{array}$} & 1,0062 \\
37,5 & $-1,7661$ & 0,5422 & 0,68 \\
50 & $-0,6443$ & 0,5007 & 0,29 \\
62,5 & $-0,5467$ & 0,6258 & 0,47 \\
75 & $-0,8457$ & 0,2609 & 0,15 \\
Conj. & 0,0375 & 0,5214 & 0,21
\end{tabular}

Overall

\begin{tabular}{|c|c|c|c|}
\hline \multicolumn{4}{|c|}{ Phosphorus } \\
\hline 25 & $-1,7635$ & 0,8847 & 0,62 \\
\hline 37,5 & $-1,9854$ & 0,9759 & 0,87 \\
\hline 50 & $-1,9684$ & 0,9685 & 0,90 \\
\hline 62,5 & $-1,9548$ & 0,9636 & 0,86 \\
\hline 75 & $-1,2345$ & 0,6656 & 0,83 \\
\hline Conj. & $-1,6967$ & 0,8585 & 0,61 \\
\hline \multicolumn{4}{|c|}{ Magnésio (kg) } \\
\hline 25 & $-2,7773$ & 0,8044 & 0,37 \\
\hline 37,5 & $-2,2263$ & 0,5762 & 0,23 \\
\hline 50 & $-1,8833$ & 0,4335 & 0,15 \\
\hline 62,5 & $-1,8263$ & 0,4112 & 0,17 \\
\hline 75 & $-1,3918$ & 0,2317 & 0,07 \\
\hline Conj. & $-1,8711$ & 0,4325 & 0,13 \\
\hline Overall & & & \\
\hline \multirow{2}{*}{\multicolumn{4}{|c|}{$\begin{array}{c}\text { Potássio (kg) } \\
\text { Potassium }\end{array}$}} \\
\hline & & & \\
\hline 25 & $-3,3086$ & 1,2816 & 0,85 \\
\hline 37,5 & $-3,3286$ & 1,2897 & 0,85 \\
\hline 50 & $-3,1784$ & 1,2284 & 0,86 \\
\hline 62,5 & $-2,6096$ & 0,9930 & 0,80 \\
\hline 75 & $-2,7329$ & 1,0435 & 0,82 \\
\hline Conj. & $-2,8994$ & 1,1155 & 0,79 \\
\hline Overall & & & \\
\hline
\end{tabular}

(K) no corpo vazio, em função do logaritmo do peso do corpo vazio (PCVZ), obtidos para cada nível de concentrado nas dietas e para todos os tratamentos em conjunto. Como o teste de identidade de modelos aplicado às equações de regressão do logaritmo do conteúdo corporal dos elementos minerais, em fun- ção do logaritmo do PCVZ, para os cinco níveis de concentrado na dieta, indicou não haver diferença entre os tratamentos, foram utilizadas as equações relativas aos dados em conjunto.

Os coeficientes de determinação $\left(\mathrm{r}^{2}\right)$ das equações de regressão, especialmente para $\mathrm{Ca}, \mathrm{P}$ e $\mathrm{Mg}$, de modo geral, não mostraram bom ajustamento das equações aos dados, como pode ser verificado por VÉRAS et al. (2000b) para gordura, proteína e energia. Contudo, este comportamento também foi verificado por FONTES (1995), em compilação de dados de vários experimentos conduzidos no Brasil, e por PAULINO (1996), com zebuínos, cuja justificativa, possivelmente, seja devido às quantidades de minerais relativamente pequenas, em comparação ao PCVZ. Também, os animais do presente trabalho se encontravam em fase de engorda em confinamento, o que, para COELHO DA SILVA (1995), seria uma etapa em que, principalmente, as deposições de $\mathrm{Ca}$ e $\mathrm{P}$ seriam muito baixas, visto que a fase mais intensa de crescimento já havia sido concluída.

Os conteúdos de $\mathrm{Ca}, \mathrm{P}, \mathrm{Mg}$ e $\mathrm{K}$ por kg de ganho de PCVZ, ou seja, as exigências líquidas destes minerais para ganho de um kg de PCVZ são apresentadas na Tabela 2.

Os resultados demonstraram diminuição nas concentrações de $\mathrm{Ca}, \mathrm{P}$ e $\mathrm{Mg}$, com o aumento do peso vivo (PV), o que era esperado, já que VÉRAS et al. (2000b) observaram aumento da gordura corporal com a elevação do PV para todos os níveis de concentrado nas dietas e, segundo COELHO DA SILVA (1995) e FONTES (1995), quanto maior a deposição de gordura, menores os depósitos de minerais; além do fato de o tecido adiposo geralmente não conter $\mathrm{Ca}$.

Os valores observados no presente trabalho são inferiores a $14 \mathrm{~g} / \mathrm{kg}$ de ganho de $\mathrm{PV}$, preconizado pelo ARC (1980), como exigência líquida diária de Ca para ganho de um quilograma. Para um animal pesando $400 \mathrm{~kg}$ de PV e ganhando um $\mathrm{kg} / \mathrm{dia}$, os resultados também são inferiores aos relatados pelo AFRC (1991), de 11,9 g/dia.

$\mathrm{O}$ decréscimo dos requisitos de $\mathrm{Ca}, \mathrm{P}$ e $\mathrm{Mg}$, são coerentes com vários relatos na literatura, entre eles os de FONTES (1995), em análise de vários trabalhos; PAULINO et al. (1997), com zebuínos; e FERREIRA et al. (1998a,b), que utilizaram bovinos F1 Simental-Nelore, não-castrados, alimentados com dietas semelhantes às do presente trabalho.

O AFRC (1991) também observou redução nas 
1110 Rev. bras. zootec.

Tabela 2 - Exigências líquidas de cálcio $(\mathrm{Ca})$, fósforo $(P)$, magnésio (Mg) e potássio (K), em $\mathrm{g} / \mathrm{kg}$ GPCVZ, de bovinos Nelore, em função do peso vivo (PV) ou do PCVZ

Table 2 - Calcium, phosphorus, magnesium and potassium liquid requirements, in $\mathrm{g} / \mathrm{kg}$ EBWG, of Nellore bulls, in function of live weight (LW) or EBWG

$\overline{\mathrm{PV}(\mathrm{kg})} \quad \mathrm{PCVZ}(\mathrm{kg}) \quad$ Exigências líquidas (g/kg GPCVZ)

\begin{tabular}{|c|c|c|c|c|c|}
\hline \multirow[t]{2}{*}{$L W$} & \multirow[t]{2}{*}{$E B W G$} & \multicolumn{4}{|c|}{ Dietary requirements $(\mathrm{g} / \mathrm{kg} E B W G)$} \\
\hline & & $\mathrm{Ca}$ & $\mathrm{P}$ & $\mathrm{Mg}$ & K \\
\hline 300 & 251,07 & 9,60 & 7,90 & 0,25 & 2,66 \\
\hline 350 & 300,62 & 8,80 & 7,70 & 0,23 & 2,72 \\
\hline 400 & 350,18 & 8,18 & 7,53 & 0,21 & 2,77 \\
\hline 450 & 399,73 & 7,68 & 7,39 & 0,19 & 2,81 \\
\hline
\end{tabular}

concentrações de $\mathrm{Ca}$ e $\mathrm{P}$ por $\mathrm{kg}$ de ganho com a elevação do peso corporal, cuja justificativa seria devido ao aumento no teor de gordura corporal, já discutido anteriormente, e concomitante redução na proporção de ossos verificada com o aumento do peso e da idade dos animais. A redução na proporção de ossos no corpo do animal induz queda nas concentrações de Ca e P, já que os ossos contêm cerca de $99 \%$ do $\mathrm{Ca}$ e de $80 \%$ do $\mathrm{P}$ corporais.

A exigência líquida de $\mathrm{Ca}$, para um animal de 400 $\mathrm{kg}$ de PV , foi de $8,18 \mathrm{~g}$. Este valor é bastante próximo a $8,63 \mathrm{~g}$, obtidos por PAULINO et al. (1997), e 20,81\% inferior ao relatado por FERREIRA et al. (1998a), para o mesmo PV. Já em relação ao $\mathrm{P}$, os requisitos líquidos de um animal pesando $400 \mathrm{~kg}(7,53 \mathrm{~g})$ foram quase $100 \%$ superiores aos observados pelos referidos autores, porém mais próximos do relatado por FONTES (1995), de 6,87, para animais não-castrados.

Vale salientar que os valores obtidos no presente trabalho são superiores aos observados por FONTES (1995) para animais castrados, confirmando que estes últimos depositam gordura mais precocemente, aumentando o teor de gordura corporal, com consequente diluição do conteúdo corporal de minerais e redução dos respectivos requisitos líquidos.

Utilizando-se o modelo matemático proposto pelo AFRC (1991) para um bovino com $400 \mathrm{~kg}$ de PV e, considerando-se peso à maturidade de $450 \mathrm{~kg}$, os requerimentos líquidos estimados para $\mathrm{Ca}$ e $\mathrm{P}$ por $\mathrm{kg}$ de ganho seriam de 10,09 e 5,96 g, respectivamente. Os valores obtidos para Ca são inferiores, e os para $\mathrm{P}$, superiores aos estimados conforme metodologia proposta pelo referido conselho. COELHO DA SILVA (1995), comparando dados obtidos no Brasil com os estimados segundo metodologia preconizada pelo
AFRC (1991), concluiu que as estimativas das exigências líquidas de $\mathrm{Ca}$ e $\mathrm{P}$ diferiram em torno de $100 \%$ daquelas propostas pelo referido conselho.

Utilizando-se os requisitos líquidos de proteína obtidos por VÉRAS et al. (2000b), para um animal pesando $400 \mathrm{~kg}$, e as exigências líquidas de Ca e P obtidos no presente trabalho, as relações $\mathrm{g} \mathrm{Ca} / 100 \mathrm{~g}$ de proteína retida e $\mathrm{g} / 100 \mathrm{~g}$ de proteína retida foram iguais a 5,17 e 4,76, respectivamente. Estes valores diferiram dos preconizados pelo NRC (1996), que são iguais a 7,10 e 3,90, respectivamente.

As exigências líquidas de $\mathrm{Mg}$ verificadas no presente trabalho são inferiores às estimadas pelo ARC (1980) e as relatadas por FERREIRA et al. (1998b), porém similares as encontradas por PAULINO et al. (1997), para zebuínos. Já em relação aos resultados obtidos para $\mathrm{K}$, estes foram mais elevados que o predito pelo ARC (1980) e os obtidos por PAULINO et al. (1997), FERREIRA et al. (1998b), entre outros.

A partir dos coeficientes médios de absorção aparente determinados por VÉRAS et al. (2000a), de 53,13; 37,33; 39,62; e 65,54\%, para Ca, P, Mg e K, respectivamente, e das estimativas das exigências líquidas para ganho, foram estimados os requisitos dietéticos de $\mathrm{Ca}, \mathrm{P}, \mathrm{Mg}$ e $\mathrm{K}$, por $\mathrm{kg}$ de ganho de PCVZ (Tabela 3), o que corresponde às exigências para ganho de um kg de PV já que o fator de conversão dos requerimentos de GPCVZ em ganho de PV, obtido por VÉRAS et al. (2000b), foi igual a um.

As exigências dietéticas de $\mathrm{Ca}$ e $\mathrm{P}$ para um animal com $400 \mathrm{~kg}$ de PV, ganhando um kg por dia, diferiram das preditas pelo NRC (1996), que são de 19 e 8 g/dia, respectivamente. Vale ressaltar que os requerimentos obtidos no presente trabalho foram determinados utilizando-se o coeficiente médio de absorção aparente determinado previamente por VÉRAS et al. (2000a), e não a partir de relatos na literatura.

Tabela 3 - Exigências dietéticas de cálcio $(\mathrm{Ca})$, fósforo $(P)$, magnésio $(\mathrm{Mg})$ e potássio $(\mathrm{K})$, em g/kg GPCVZ, de bovinos Nelore, em função do peso vivo (PV) ou do PCVZ

Table 3 - Calcium, phosphorus, magnesium and potassium dietary requirements, in $\mathrm{g} / \mathrm{kg}$ EBWG, of Nellore bulls, in function of the live weight (LW) or EBWG

$\mathrm{PV}(\mathrm{kg}) \quad \mathrm{PCVZ}(\mathrm{kg}) \quad$ Exigências líquidas (g/kg GPCVZ)

LW EBWG Dietary requirements $(\mathrm{g} / \mathrm{kg} E B W G)$

\begin{tabular}{cccccc} 
& & $\mathrm{Ca}$ & $\mathrm{P}$ & $\mathrm{Mg}$ & $\mathrm{K}$ \\
\cline { 3 - 6 } & & 18,07 & 21,16 & 0,63 & 4,06 \\
300 & 251,07 & 16,56 & 20,63 & 0,58 & 4,15 \\
400 & 300,62 & 15,40 & 20,17 & 0,53 & 4,23 \\
450 & 350,18 & 14,46 & 19,80 & 0,48 & 4,29
\end{tabular}




\section{Conclusões}

As exigências líquidas de $\mathrm{Ca}$ foram menores e as de $\mathrm{P}$, maiores, que as preconizadas pelo AFRC (1991) e pelo NRC (1996).

Os requerimentos líquidos de $\mathrm{Mg}$ foram inferiores e os de K, superiores, aos relatados pelo ARC (1980).

\section{Referências Bibliográficas}

AGRICULTURAL ANDFOOD RESEARCHCOUNCIL - AFRC. 1991. Technical committee on responses to nutrients, Report 6. A reappraisal of the calcium and phosphorous requirements of sheep and cattle. Nut. Abs. Rev., 61(9):576-612.

AGRICULTURAL RESEARCH COUNCIL - ARC. 1980. The nutrient requirements of ruminants livestock. London: Commonwealth Agricultural Bureaux. 351p.

COELHO DA SILVA, J.F. Exigências de macroelementos inorgânicos para bovinos: o sistema ARC/AFRC e a experiência no Brasil. In: SIMPÓSIO INTERNACIONAL SOBRE EXIGÊNCIAS NUTRICONAIS DE RUMINANTES, 1995, Viçosa, MG. Anais... Viçosa, MG: DZO, 1995. p. 467-504. COMMONWEALTH SCIENTIFIC AND INDUSTRIAL RESEARCH ORGANIZATION - CSIRO. 1990. Feeding standards for Australian livestock - ruminants. Victoria: Australia Agricultural Council. 266p.

DAYRELL, M.S. Suplementação mineral para vacas de leite de alta produção. In: MINI-SIMPÓSIO DO COLÉGIO BRASILEIRO DE NUTRIÇÃO ANIMAL. NUTRIÇÃO E ALIMENTAÇÃO DE GADO LEITEIRO, 9., 1993, Valinhos. Anais... Campinas: C.B.N.A, 1993. p.71-81.

FERREIRA, M.A., VALADARES FILHO, S.C., VALADARES, R.F.D. et al. Composição corporal e exigências, de cálcio e fósforo para ganho de peso de bovinos $\mathrm{F}_{1}$ Simental x Nelore, não castrados. In: REUNIÃO ANUAL DA SOCIEDADE BRASILEIRA DE ZOOTECNIA, 35., 1998, Botucatu. Anais... Botucatu, SP: SBZ, 1998a. p.389-391.

FERREIRA, M.A., VALADARES FILHO, S.C., VALADARES, R.F.D. et al. Composição corporal e exigências de magnésio, sódio e potássio para ganho de peso de bovinos $\mathrm{F}_{1}$ Simental $x$ Nelore, não castrados. In: REUNIÃO ANUAL DA SOCIEDADE BRASILEIRA DE ZOOTECNIA, 35., 1998, Botucatu. Anais... Botucatu, SP: SBZ, 1998b. p.392-394.

FONTES, C.A.A. Composição corporal, exigências líquidas de nutrientes para ganho de peso e desempenho produtivo de animais zebuínos e mestiços europeu-zebu. Resultados experimentais. In: SIMPÓSIO INTERNACIONAL SOBRE EXIGÊNCIAS NUTRICIONAIS DE RUMINANTES, 1995, Viçosa, MG. Anais... Viçosa, MG: DZO, 1995. p.419-455.
1111

LENG, R.A. 1990. Factors affecting the utilization of "poorquality" forrages by ruminants particularly under tropical conditions. Nut. Res. Rev., 3(3):277-303.

NATIONAL RESEARCH COUNCIL - NRC. 1984. Nutrient requeriments of beef cattle. 6.ed. Washington, D.C. 90p.

NATIONAL RESEARCH COUNCIL - NRC. 1996. Nutrients requirements of beef cattle. 7.ed. Washington, D.C. 242p.

NICODEMO, M.L.F. Diagnóstico de deficiências minerais em bovinos. In: SIMPÓSIO GOIANO SOBRE PRODUÇÃO DE BOVINOS DE CORTE. 1999, Goiânia, GO. Anais...Goiânia, GO: CBNA, 1999, p.57-80.

PAULINO, M.F. Composição corporal e exigências de energia, proteína e macroelementos minerais ( $\mathrm{Ca}, \mathrm{P}, \mathrm{Mg}$, $\mathrm{Na}$ e $\mathrm{K}$ ) de bovinos de quatro raças zebuínas em confinamento. Viçosa, MG: UFV, 1996. 80 p. Tese (Doutorado em Zootecnia) Universidade Federal de Viçosa, 1996.

PAULINO, M.F., FONTES, C.A.A., JORGE, A.M. et al. Exigências de macroelementos minerais ( $\mathrm{Ca}, \mathrm{P}, \mathrm{Mg}, \mathrm{Na}$ e $\mathrm{K}$ ) para ganho de peso de bovinos não castrados de quatro raças zebuínas. In: REUNIÃO ANUAL DA SOCIEDADE BRASILEIRA DE ZOOTECNIA, 34., 1997. Juiz de Fora, MG. Anais ... Juiz de Fora: SBZ, 1997, p.19-21.

SPEARS, J.W. Minerals in forage. In: FAHEY JR., G.C. (Ed.) Forage quality, evaluation and utilization. American Society of Agronomy. NATIONAL CONFERENCE ON FORAGE QUALITY, EVALUATION AND UTILIZATION, 1994. p.281-317.

VÉRAS, A.S.C., VALADARES FILHO, S.C.V., COELHO DA SILVA, J.F. et al. 2000a. Consumo e digestibilidade aparente em bovinos Nelore, não-castrados, alimentados com rações contendo diferentes níveis de concentrado. Rev. bras. zootec., 29(6):2367-2378 (supl. 2).

VÉRAS, A.S.C., VALADARES FILHO, S.C., COELHO DA SILVA, J.F. et al. 2000b. Composição corporal e requisitos energéticos e protéicos de bovinos Nelore, não-castrados, alimentados com rações contendo diferentes níveis de concentrado. Rev. bras. zootec., 29(6):2379-2389 (supl. 2).

Recebido em: 01/12/99

Aceito em: 21/02/01 